\title{
On the rate of convergence to the neutral attractor of a family of one-dimensional maps
}

\author{
by \\ T. Nowicki, M. Sviridenko, G. Świrszcz and \\ S. Winograd (Yorktown Heights, NY)
}

To Michat Misiurewicz on his 60th birthday

Abstract. For a family of maps

$$
f_{d}(p)=1-(1-p / d)^{d}, \quad d \in[2, \infty], p \in[0,1] .
$$

we analyze the speed of convergence (including constants) to the globally attracting neutral fixed point $p=0$. The study is motivated by a problem in the optimization of routing.

The aim of this paper is twofold: (1) to extend the usage of dynamical systems to unexplored areas of algorithms and (2) to provide a toolbox for a precise analysis of the iterates near a non-degenerate neutral fixed point.

1. Reaching out. Dynamical systems initiated by Henri Poincaré in [9] were first developed for the study of physical systems described by (ordinary) differential equations, then evolved to include discrete iterations, partial differential equations, then by the concept of ergodicity to probabilistic systems including some number theory problems, by the notion of partitions to symbolic systems, and by extending the concept of time to (sub)groups to lattices and other algebraic systems. Whenever someone speaks of evolution of sorts the dynamical systems people get excited and start looking for new openings.

One such opening, not too crowded yet, is the area of diverse and interesting problems in algorithms and data structures, say in random graphs theory. Problems arise when the construction is made of layers and the phenomena on the next layer depend on the previous one. The questions include estimating probabilities, weights of specific subgraphs, reconstruction

2010 Mathematics Subject Classification: 37E05, 34D45, 90B80.

Key words and phrases: neutral attractor, assignment problem, dynamics in algorithms. 
of trees, optimizing weights and modeling optimal connections with recursive algorithms [4,5].

From the ivory tower vantage point these problems are finite and usually there is an existential proof of a solution and thus they present no motivation for further studies. But the existence of a solution and its effectiveness in practice are quite different issues. We present here a technical toolbox which can be used in estimating the speed of convergence of the surviving probability of a particle in a random graph, a problem which evolved from a heuristic optimization of an assignment problem.

In this context an evolution equation appears which leads to a globally attracting fixed point. This could kill any dynamical appeal, but the attractor is neutral and the problem of the speed of convergence, including constants, may be of a practical interest. Strong and robust tools exist for hyperbolic cases, but the structurally unstable neutral situation which marks a transition from one stable regime to another is more subtle. In such cases there is an interest in considerable accuracy of the rate of convergence/divergence which includes the estimates of the constants involved in the asymptotic behavior.

1.1. Neutral attractors. The aim of this paper is to analyze the behavior of the sequences which are given recursively as iterations of the functions with a neutral fixed point. More precisely we consider the family of functions $f_{d}: \mathbb{R}^{+} \rightarrow[0,1]$ given by

$$
f_{d}(p)=1-(1-p / d)^{d}, \quad d \geq 2, p \in[0, d) .
$$

The case $d=\infty$ is included as $f_{\infty}(p)=1-\exp (-p)$, and in all subsequent results in this case one takes first the limit $d \rightarrow \infty$ and then performs the calculations.

An isolated neutral (non-hyperbolic) fixed point $x$ of a map $f$ fulfills two conditions: first $f(x)=x$ and then $\left|f^{\prime}(x)\right|=1$ (in higher dimensions one may require that the spectrum of $D f(x)$ lies on the unit circle). As opposed to hyperbolic repellers, attractors and saddles, the behavior of the iterates near a neutral point is governed not by the linear approximation (value of the derivative at the fixed point) but by higher order terms in the expansion of the map. In particular, the speed of escape or convergence in the neighborhood of a neutral fixed point is not exponential but rather polynomial in nature. In a typical situation when the difference equation is $x_{n+1}=x_{n}-x_{n}^{m}$ for $m>1$ we have $x_{n} \approx(a+b n)^{-1 /(m-1)}$, which follows from the comparison with the differential equation $d x=-x^{m} d t$.

The study of neutral fixed points is not very much developed. They are considered non-typical, in the sense that a small general perturbation of the map makes them hyperbolic. There is a classification of neutral points for holomorphic maps of the complex plane [7], some sporadic results in low (1, 
2 and 3) dimensional dynamics and some results concerning the saddle-node bifurcations and funneling effects for a class of differential equations [1].

For the family of maps $f_{d}$ in this paper we were motivated by an optimization problem, which we will sketch in the Appendix, which deals with particles competing for a route in a special layered graph and the probabilities of a particle to arrive in the $k$ th layer. There is a way to solve this optimization, which leads to a bound of order $2 /(k+1)$. Our intention is make the bound more precise and to pave the way for a broader approach, useful hopefully in other classes of problems as well, where the values of interest form a sequence of iterates of the map $f_{d}$.

1.2. Main result. For $d \geq 2$ and $p \in[0, d]$ define

$$
f_{d}(p)=1-(1-p / d)^{d},
$$

and with $p_{-1}=d$ and $p_{0}=1$,

$$
p_{k}=f_{d}\left(p_{k-1}\right) .
$$

Our main result is to provide the following bounds:

THEOREM.

(1) For $k \geq 1$ and $d \in\{2,3\} \cup[4, \infty]$ we have

$$
\left(1-\frac{3}{\ln k+1}\right) \frac{\alpha}{k+\beta} \leq p_{k} \leq \frac{\alpha}{k+\beta} .
$$

(2) There exists a positive bounded (uniformly in $d$ ) sequence $\epsilon_{k}$ such that

$$
p_{k}=\frac{\alpha}{k+\epsilon_{k} \ln k} \text {. }
$$

Here

$$
\begin{aligned}
& \alpha=\alpha(d)=\frac{2 d}{d-1}, \\
& \beta=\beta(d)=\frac{d+1}{d-1}, \quad \beta=\alpha-1,
\end{aligned}
$$

and for later use define

$$
\gamma=\gamma(d)=\frac{3 d}{d-2}, \quad d>2 .
$$

REMARK 1.1. We shall need the Taylor expansion up to the third term only. The argument we are using works for $d=2,3$ (finite expansions) or for $d \geq 4$ (to have a good remainder estimate). We will skip the details for $d \in(2,3) \cup(3,4)$.

Our approach can be applied to any $\mathcal{C}^{3}$ function which satisfies conditions (2.1) and (2.2) below with positive $\alpha$ and $\gamma$, but the statement of the results would have to be reformulated carefully to indicate the role of the constants. 


\section{Basic properties of iterations and technical lemmata}

2.1. Basic properties of $f_{d}$. For each $2 \leq d \leq \infty$ the map $f_{d}$ is positive on $(0, d)$ and for every integer $1 \leq m \leq d$ the $m$ th derivative $f^{(m)}(p)$ has a constant sign $(-1)^{m+1}$ on $(0, d)$. The following properties follow immediately by calculation:

(1) $f_{d}(0)=0, f_{d}(d)=1, f_{d}^{\prime}(0)=1$.

(2) Define

$$
\begin{aligned}
\mathcal{O}_{m}(p) & =\left|\frac{f^{(m)}(p)}{m !}\right|=\frac{1}{m !}\left|(-1)^{m+1}\left(1-\frac{p}{d}\right)^{d-m} \prod_{i=0}^{m-1} \frac{d-i}{d}\right| \\
& =\frac{6}{m !}\left(1-\frac{p}{d}\right)^{d-3} \frac{d-1}{2 d} \frac{d-2}{3 d} \prod_{i=3}^{m-1} \frac{d-i}{d-p} .
\end{aligned}
$$

Then for $p \in(0,1)$ we have $\mathcal{O}_{m}(p) \in\left(0, \frac{6}{\alpha \gamma m !}\right)$. In particular, for $m=4$ we have

$$
\mathcal{O}_{m}(p)=\frac{1}{4 \alpha \gamma}\left(1-\frac{p}{d}\right)^{d-3} \frac{d-3}{d-p} \geq \frac{d-3}{4 d} \frac{1}{\alpha \gamma} e^{-p} .
$$

We have used $e^{x} \geq 1+x$ with $x=p /(d-p)$.

(3) For $0<m<d, f_{d}^{(m)}(d)=0$.

(4) By Taylor expansion, for some $\xi \in(0, p)$,

$$
\begin{aligned}
f_{d}(p) & =\sum_{i=1}^{m-1}(-1)^{i+1} \frac{p^{i}}{i !} \prod_{j=0}^{i-1}\left(1-\frac{j}{d}\right)+\frac{f^{(m)}(\xi)}{m !} p^{m} \\
& =\sum_{i=1}^{m-1}(-1)^{i+1} \frac{p^{i}}{i !} \prod_{j=1}^{i-1}\left(1-\frac{j}{d}\right)+(-1)^{m+1} \mathcal{O}_{m}(\xi) p^{m}
\end{aligned}
$$

where $\mathcal{O}_{m}(\xi)<\mathcal{O}_{m}(p)$.

(5) For $p \in(0, d)$,

$$
\begin{array}{ll}
f_{d}(p) \leq \sum_{i=1}^{m-1}(-1)^{i+1} \frac{p^{i}}{i !} \prod_{j=0}^{i-1}\left(1-\frac{j}{d}\right) & \text { for even } m, \\
f_{d}(p) \geq \sum_{i=1}^{m-1}(-1)^{i+1} \frac{p^{i}}{i !} \prod_{j=0}^{i-1}\left(1-\frac{j}{d}\right) & \text { for odd } m .
\end{array}
$$

(6) In particular, as $d \geq 2$, for $0<p<d$, using (1.1) and (1.2) we have $f_{d}(p)<p$ and 
(2.1) $f_{d}(p) \geq p-\frac{d-1}{2 d} p^{2}=p-\frac{p^{2}}{\alpha}=: l(p)$,

$$
f_{d}(p) \leq p-\frac{d-1}{2 d} p^{2}+\frac{(d-1)(d-2)}{6 d^{2}} p^{3}=p-\frac{p^{2}}{\alpha}+\frac{p^{3}}{\alpha \gamma}=: u(p) .
$$

Obviously $f_{d}([0, d])=[0,1]$ and as $f_{d}$ is increasing we can trap each element of the trajectory of any point between two consecutive elements of the trajectory of $p=1$.

Lemma 2.1 (Technicalities 1 ). For all $d \in(1, \infty]$ and $k \geq 1$,

$$
\begin{aligned}
& \frac{1}{k+\beta}-\frac{1}{(k+\beta)^{2}}+\frac{\alpha}{\gamma} \frac{1}{(k+\beta)^{3}} \leq \frac{1}{k+1+\beta}, \quad \text { or in other words, } \\
& \frac{1}{k+\frac{d+1}{d-1}}-\frac{1}{\left(k+\frac{d+1}{d-1}\right)^{2}}+\frac{2(d-2)}{3(d-1)} \frac{1}{\left(k+\frac{d+1}{d-1}\right)^{3}} \leq \frac{1}{k+1+\frac{d+1}{d-1}} .
\end{aligned}
$$

Proof. Define

$$
\Delta=\Delta_{k}(d)=k+\beta=k+\frac{d+1}{d-1} .
$$

As we have

$$
\frac{1}{1+\Delta}-\frac{1}{\Delta}+\frac{1}{\Delta^{2}}=\frac{\Delta^{2}-\Delta(1+\Delta)+(1+\Delta)}{\Delta^{2}(1+\Delta)}=\frac{1}{\Delta^{2}(1+\Delta)},
$$

we need to prove that

$$
\frac{2(d-2)}{3(d-1)} \frac{1}{\Delta^{3}} \leq \frac{1}{\Delta^{2}(1+\Delta)}
$$

that is,

$$
3(d-1) \Delta-2(d-2)(\Delta+1)=(d+1) \Delta-2(d-2) \geq 0 .
$$

Indeed,

$$
\begin{aligned}
(d+1) \Delta-2(d-2) & =\frac{\left(d^{2}-1\right) k+(d+1)^{2}-2(d-2)(d-1)}{d-1} \\
& =\frac{(k-1)\left(d^{2}-1\right)+8(d-1)+3}{d-1}>0
\end{aligned}
$$

as $k \geq 1$ and $d>1$.

For the second bound we want to estimate $1 / f(p)$ by $1 / p+1 / \alpha+b(p) p$ with a bounded $b(p)$ which we define accordingly:

$$
b(p)=\frac{1}{p}\left(\frac{1}{f(p)}-\frac{1}{p}-\frac{1}{\alpha}\right) .
$$


Lemma 2.2 (Further approximation). The function $b(p)$ is positive and bounded away from 0 and infinity uniformly in $p \in[0,1]$ and $d \geq 2$. In particular, the limit $b(0)>0$ exists.

Proof. We use Taylor expansion (2.1) and the definition (2.2) of $u(p)$ : $f(p)=u(p)-\mathcal{O} p^{4}$ for $\mathcal{O}=\mathcal{O}_{4}(\xi) \in\left(\frac{d-3}{4 d} \frac{1}{\alpha \gamma} e^{-p}, \frac{1}{4 \alpha \gamma}\right)$. We have

$$
\begin{aligned}
b(p) & =\frac{1}{\alpha p^{2} f(p)}(\alpha p-(\alpha+p) f(p)) \\
& =\frac{1}{\alpha p^{2} f(p)}\left(\alpha p-(\alpha+p)\left(p-\frac{p^{2}}{\alpha}+\frac{p^{3}}{\alpha \gamma}-p^{4} \mathcal{O}\right)\right) \\
& =\frac{1}{\alpha p^{2} f(p)}\left(\alpha p-\alpha p+p^{2}-\frac{p^{3}}{\gamma}-p^{2}+\frac{p^{3}}{\alpha}+\left(\alpha \mathcal{O}-\frac{1}{\alpha \gamma}\right) p^{4}+\mathcal{O} p^{5}\right) \\
& =\frac{1}{\alpha p^{3}\left(1-\frac{p}{\alpha}+\frac{p^{2}}{\alpha \gamma}-\mathcal{O} p^{3}\right)} p^{3}\left(\frac{(\gamma-\alpha)-p}{\alpha \gamma}+\alpha \mathcal{O} p+\mathcal{O} p^{2}\right) .
\end{aligned}
$$

As $\gamma-\alpha=\frac{d(d+1)}{(d-1)(d-2)} \geq 1$ that shows that $b(p)>0$ for all $p$. From the bounds on $\mathcal{O}$ we get

$$
\begin{aligned}
\frac{1}{336} & <\frac{(\gamma-\alpha)-p+\frac{d-3}{4 d} e^{-p} p(\alpha+p)}{\alpha\left(\alpha \gamma-\gamma p+p^{2}-\frac{d-3}{4 d} e^{-p} p^{3}\right)}<b(p) \\
& <\frac{(\gamma-\alpha)-p+\frac{\alpha p+p^{2}}{4}}{\alpha\left(\alpha \gamma-\gamma p+p^{2}-\frac{p^{3}}{4}\right)}<\frac{5}{6}
\end{aligned}
$$

Numerical estimations are closer to $1 / 30 \leq b(p) \leq 1 / 8$.

Lemma 2.3 (Technicalities 2). For $q_{0}>0, a>0$ and a bounded sequence $b_{n}>0$ define

$$
q_{n+1}=q_{n}+a+b_{n} / q_{n} .
$$

Then there exists a bounded sequence $c_{n}>0$ such that

$$
q_{n}=n a+c_{n} \ln \left(1+n a / q_{0}\right) .
$$

Proof. Let $b<b_{n}<B$. We have $q_{n+1}>q_{n}+a$ and hence $q_{n}>q_{0}+n a$. Thus

$$
\sum_{k<n} \frac{b_{k}}{q_{k}}<B \sum_{k<n} \frac{1}{q_{0}+k a}<B \ln \frac{q_{0}+n a}{q_{0}} .
$$

Hence

$$
q_{n}=q_{0}+n a+\sum_{k \leq n} \frac{b_{k}}{q_{k}} \leq q_{0}+n a+B \ln \frac{q_{0}+n a}{q_{0}} .
$$


We can use it again to get

$$
\begin{aligned}
q_{n} & >q_{0}+n a+\sum_{k<n} \frac{b_{k}}{q_{0}+k a+B \ln \frac{q_{0}+k a}{q_{0}}} \\
& >q_{0}+n a+\sum_{k<n} b_{k}\left(\frac{1}{q_{0}+k a+B \ln \frac{q_{0}+k a}{q_{0}}}-\frac{1}{q_{0}+k a}\right)+\sum_{k<n} \frac{b_{k}}{q_{0}+k a} \\
& \geq q_{0}+n a+b \ln \frac{q_{0}+(n-1) a}{q_{0}}-\sum_{k<\infty} \frac{B \ln \frac{q_{0}+k a}{q_{0}}}{\left(q_{0}+k a+B \ln \frac{q_{0}+k a}{q_{0}}\right)\left(q_{0}+k a\right)} \\
& \geq q_{0}+n a+b \ln \frac{q_{0}+(n-1) a}{q_{0}}-\text { const. }
\end{aligned}
$$

That means that the sequence $c_{n}=\left(q_{n}-q_{0}-n a\right) / \ln \left(1+\frac{a}{q_{0}} n\right)$ is bounded. We adjust it to cover small $n$ s where the last estimate may be negative.

3. The bounds in the Theorem. Fix $d$, set $p_{0}=1$ and define $p_{k+1}=$ $f_{d}\left(p_{k}\right)$ for integers $0 \leq k<d$. As $0<f(p)<p<d$ the sequence $p_{k}$ is decreasing. We are interested in the asymptotic behavior of $p_{k}$ as $k \rightarrow d$ and $d \rightarrow \infty$

First we prove:

Proposition 3.1 (The upper bound). For $d \geq 2$,

$$
p_{k} \leq \frac{\alpha}{k+\beta} .
$$

The numbers $\alpha$ and $\beta$ were defined in (1.1) and (1.2).

Proof. Let

$$
u(p)=u_{d}(p)=p-\frac{d-1}{2 d} p^{2}+\frac{(d-1)(d-2)}{6 d^{2}} p^{3}
$$

if $d<\infty$, and $u_{\infty}(p)=p-\frac{1}{2} p^{2}+\frac{1}{6} p^{3}$. For $p \leq 1$ the function $u$ is increasing as $u^{\prime}(p)>1-(d-1) p / d \geq 1 / d$. (In fact, for $d \geq 3$ we have $u^{\prime}(p) \geq 0$ for all $p$.) By $(2.2)$ we have $f(p)<u(p)$ for $0<p<d$. Let $u_{0}=1$ and $u_{k+1}=u\left(u_{k}\right)$. Then inductively $p_{k} \leq u_{k}$, as

$$
p_{k+1}=f\left(p_{k}\right) \leq u\left(p_{k}\right) \leq u\left(u_{k}\right)=u_{k+1} .
$$

It is therefore enough to prove $u_{k} \leq \frac{\alpha}{k+\beta}$. We check for $k=0$ and $k=1$. First we get $u_{0}=1 \leq \frac{2 d}{d+1}=\frac{\alpha}{0+\beta}$, as $d \geq 1$. Then

$$
\begin{aligned}
u_{1} & =u\left(u_{0}\right)=1-\frac{d-1}{2 d}+\frac{(d-1)(d-2)}{6 d^{2}}=\frac{6 d^{2}-3 d(d-1)+(d-1)(d-2)}{6 d^{2}} \\
& =\frac{2 d^{2}+1}{3 d^{2}}<1=\frac{2 d}{d-1+d+1}=\frac{\alpha}{1+\beta} .
\end{aligned}
$$


Next for $k \geq 1$ we use induction and Lemma 2.1:

$$
\begin{aligned}
u_{k+1}= & u\left(u_{k}\right) \leq u\left(\frac{\alpha}{k+\beta}\right) \\
= & \frac{\alpha}{k+\beta}-\frac{d-1}{2 d}\left(\frac{\alpha}{k+\beta}\right)^{2}+\frac{(d-1)(d-2)}{6 d^{2}}\left(\frac{\alpha}{k+\beta}\right)^{3} \\
= & \frac{2 d}{d-1} \frac{1}{k+\frac{d+1}{d-1}}-\frac{d-1}{2 d}\left(\frac{2 d}{d-1}\right)^{2} \frac{1}{\left(k+\frac{d+1}{d-1}\right)^{2}} \\
& +\frac{(d-1)(d-2)}{6 d^{2}}\left(\frac{2 d}{d-1}\right)^{3} \frac{1}{\left(k+\frac{d+1}{d-1}\right)^{3}} \\
= & \frac{2 d}{d-1}\left(\frac{1}{k+\frac{d+1}{d-1}}-\frac{1}{\left(k+\frac{d+1}{d-1}\right)^{2}}+\frac{2(d-2)}{3(d-1)} \frac{1}{\left(k+\frac{d+1}{d-1}\right)^{3}}\right) \\
\leq & \alpha \frac{1}{k+1+\beta} \quad(\text { by Lemma 2.1). }
\end{aligned}
$$

Now we prove:

Proposition 3.2 (The lower bound). For $d \geq 2$,

$$
p_{k}>\left(1-\frac{3}{\ln \frac{k+2}{2}+2}\right) \frac{\alpha}{k+\beta} .
$$

Proof. Let

$$
l(p)=p-\frac{d-1}{2 d} p^{2}=p-\frac{p^{2}}{\alpha} .
$$

Then again $l(p)$ is increasing for $0 \leq p \leq 1$ and $l(p) \leq f(p)$. Define $l_{0}=1$ and $l_{k+1}=l\left(l_{k}\right)$. Then inductively $l_{k} \leq p_{k}$, as

$$
l_{k+1}=l\left(l_{k}\right) \leq l\left(p_{k}\right) \leq f\left(p_{k}\right)=p_{k+1} .
$$

Define $\mu_{k}$ by

$$
l_{k}=\mu_{k} \frac{\alpha}{k+\beta} .
$$

It suffices to prove $\mu_{k}>1-3 /\left(\ln \frac{k+2}{2}+2\right)$.

Given $\mu_{0}=\beta / \alpha=(d+1) / 2 d \in[1 / 2,3 / 4]$ we can define $\mu_{k}$ recursively:

$$
\begin{aligned}
\mu_{k+1} & =\frac{k+1+\beta}{\alpha} l_{k+1}=\frac{k+1+\beta}{\alpha}\left(\mu_{k} \frac{\alpha}{k+\beta}-\frac{1}{\alpha} \mu_{k}^{2}\left(\frac{\alpha}{k+\beta}\right)^{2}\right) \\
& =\frac{k+1+\beta}{k+\beta} \mu_{k}-\frac{k+1+\beta}{(k+\beta)^{2}} \mu_{k}^{2} .
\end{aligned}
$$

With $\Delta=k+\beta$ as in the proof of Lemma 2.1 (see (2.3)) denote by $g=g_{k}$ the quadratic function

$$
g(\mu)=\frac{\Delta+1}{\Delta} \mu-\frac{\Delta+1}{\Delta^{2}} \mu^{2}=\mu+\frac{(1-\mu) \mu}{\Delta}-\frac{\mu^{2}}{\Delta^{2}} .
$$


The sequence $\mu_{k}$ is generated by the iterations of the functions $g_{k}$, namely $\mu_{k+1}=g_{k}\left(\mu_{k}\right)$. Each function $g$ has two fixed points $\mu=0$ and $\mu=\bar{\mu}=$ $\Delta /(\Delta+1)$ and is increasing between 0 and $\Delta / 2$, which is greater than 1 for $k \geq 1$. As the derivative at $\mu=0$ is $1+1 / \delta>1$, and at $\bar{\mu}$ is $1-1 / \Delta<1$, for $0<\mu<\bar{\mu}$ we have

$$
\mu<g(\mu)<\bar{\mu},
$$

and for $\bar{\mu}<\mu<1$ we have $\bar{\mu}<g(\mu)<\mu$. We remark that for $k=0$ we have

$$
\bar{\mu}_{0}=\frac{d+1}{d-1+d+1}=\frac{d+1}{2 d}=\mu_{0} \geq \frac{1}{2},
$$

and that $\bar{\mu}_{k+1}>\bar{\mu}_{k}$ as $\Delta \mapsto \bar{\mu}_{k}=\Delta /(\Delta+1)$ is increasing and $\Delta_{k+1}=$ $\Delta_{k}+1$.

We prove that $\mu_{k} \leq \bar{\mu}_{k}$. From $\mu_{0}=\bar{\mu}_{0}$ we have $\mu_{1}=g_{0}\left(\mu_{0}\right)=g_{0}\left(\bar{\mu}_{0}\right)=$ $\bar{\mu}_{0}<\bar{\mu}_{1}$. Now we use (3.1) and inductively for $k>0$ we find $\mu_{k+1}=g_{k}\left(\mu_{k}\right)<$ $g_{k}\left(\bar{\mu}_{k}\right)=\bar{\mu}_{k}<\bar{\mu}_{k+1}$. Additionally from (3.1) we obtain $\mu_{k}<g_{k}\left(\mu_{k}\right)=\mu_{k+1}$ for $k>0$, that is, $\mu_{k}$ is increasing. In order to prove the estimate consider the differences

$$
\mu_{j+1}-\mu_{j}=\frac{\left(1-\mu_{j}\right) \mu_{j}}{\Delta_{j}}-\frac{\mu_{j}^{2}}{\Delta_{j}^{2}}
$$

By definition

$$
\Delta_{j}=j+\frac{d+1}{d-1},
$$

that is, $j+1 \leq \Delta_{j} \leq j+3$, as $d \geq 2$. The function $\mu \mapsto \mu(1-\mu)$ is decreasing for $\mu>1 / 2$, hence for $0<j<k$ we have $\mu_{k}\left(1-\mu_{k}\right)<\mu_{j}\left(1-\mu_{j}\right) \leq$ $\mu_{0}\left(1-\mu_{0}\right)<1 / 4$ and

$$
\left(1-\mu_{k}\right) \mu_{k} \ln \frac{k+2}{2} \leq\left(1-\mu_{k-1}\right) \mu_{k-1} \sum_{j=0}^{k-1} \frac{1}{j+3} \leq \sum_{j=0}^{k-1} \frac{\left(1-\mu_{j}\right) \mu_{j}}{\Delta_{j}} .
$$

On the other hand, $\mu_{j}<\bar{\mu}_{j}=\Delta_{j} /\left(\Delta_{j}+1\right)$, so

$$
\begin{aligned}
\sum_{j=0}^{k-1} \frac{\mu_{j}^{2}}{\Delta_{j}^{2}}<\sum_{j=0}^{k-1} \frac{\bar{\mu}_{j}^{2}}{\Delta_{j}^{2}} & =\sum_{j=0}^{k-1} \frac{1}{\left(\Delta_{j}+1\right)^{2}}<\sum_{j=0}^{k-1} \frac{1}{\Delta_{j}\left(\Delta_{j}+1\right)} \\
& =\frac{1}{\Delta_{0}}-\frac{1}{\Delta_{k}}<\frac{1}{\Delta_{0}} \leq 1 .
\end{aligned}
$$

All the estimates are independent of $d$. Now

$$
\mu_{k}=\mu_{0}+\sum_{j=0}^{k-1} \frac{\mu_{j}\left(1-\mu_{j}\right)}{\Delta_{j}}-\sum_{j=0}^{k-1} \frac{\mu_{j}^{2}}{\Delta_{j}^{2}}
$$


and we can use the previous estimates to obtain

$$
\begin{aligned}
& \mu_{k} \geq \frac{1}{2}+\left(1-\mu_{k}\right) \mu_{k} \ln \frac{k+2}{2}-1 \geq \frac{1}{2}\left(1-\mu_{k}\right) \ln \frac{k+2}{2}-\frac{1}{2}, \\
& \mu_{k} \geq \frac{\ln \frac{k+2}{2}-1}{\ln \frac{k+2}{2}+2}=1-\frac{3}{\ln \frac{k+2}{2}+2},
\end{aligned}
$$

which gives the desired estimate.

Propositions 3.1 and 3.2 prove the first part of the Theorem.

4. The approximation formula. Define

$$
F(q)=\frac{1}{f(1 / q)} .
$$

Then $f^{n}(p)=1 / F^{n}(1 / p)$.

Proposition 4.1 (Asymptotics of the conjugacy). For $q>1$ there exists $0<\kappa(q)<1 / 2$ such that

$$
F(q)=q+\frac{1}{\alpha}+\frac{\kappa(q)}{q} .
$$

Proof. We have to prove that $F(q)>q+1 / \alpha$ and the difference is $\mathcal{O}(1 / q)$. In other words, we have to prove that $f(1 / q)(q+1 / \alpha)<1$. By $(2.1)$ we have $f(p)<p-p^{2} / \alpha+p^{3} / \alpha \gamma$, with $p=1 / q$ and $\gamma=3 d /(d-2)$. Hence

$$
\begin{aligned}
f\left(\frac{1}{q}\right)\left(q+\frac{1}{\alpha}\right) & <\left(p-\frac{p^{2}}{\alpha}+\frac{p^{3}}{\alpha \gamma}\right)\left(\frac{1}{p}+\frac{1}{\alpha}\right) \\
& =1-\frac{p}{\alpha}+\frac{p^{2}}{\alpha \gamma}+\frac{p}{\alpha}-\frac{p^{2}}{\alpha^{2}}+\frac{p^{3}}{\alpha^{2} \gamma} \\
& =1-\frac{p^{2}}{\alpha^{2} \gamma}((\gamma-\alpha)-p)<1-\frac{p^{2}}{\alpha^{2} \gamma}(1-p)
\end{aligned}
$$

as

$$
\gamma-\alpha=\frac{d(d+1)}{(d-2)(d-1)} .
$$

Hence $\kappa(q)=q(F(q)-q-1 / \alpha)>0$ for $q>1$ (that is, $p<1$ ) as we always have $d \geq 2$. Using $f(p) \leq p$ we also establish that

$$
\begin{aligned}
\kappa(q) & =\frac{1}{p f(p)}\left(1-f(p)\left(\frac{1}{p}+\frac{1}{\alpha}\right)\right) \\
& >\frac{1}{p f(p)} \frac{p^{2}}{\alpha^{2} \gamma}(1-p) \geq \frac{1-p}{\alpha^{2} \gamma}=\frac{q-1}{q \alpha^{2} \gamma} .
\end{aligned}
$$


On the other hand, using (2.2) we have

$$
\begin{aligned}
\kappa(q) & =\frac{1}{p f(p)}\left(1-f(p)\left(\frac{1}{p}+\frac{1}{\alpha}\right)\right) \leq \frac{1}{p\left(p-p^{2} / \alpha\right)}\left(1-\left(\frac{1}{p}+\frac{1}{\alpha}\right)\left(p-\frac{p^{2}}{\alpha}\right)\right) \\
& =\frac{\alpha}{p^{2}(\alpha-p)}\left(1-\frac{p}{p \alpha^{2}}(\alpha+p)(\alpha-p)\right)=\frac{\alpha}{p^{2}(\alpha-p)} \frac{p^{2}}{\alpha^{2}}=\frac{1}{\alpha(\alpha-p)} \\
& <\frac{1}{\alpha(\alpha-1)} \leq \frac{1}{2} \quad \text { as } \alpha>2 .
\end{aligned}
$$

From Lemma 2.3 we conclude that there exists a bounded positive sequence $c_{n}$ such that:

Corollary 4.2. For $q>1$ the iterates of $F$ are bounded by

$$
F^{n}(q)=q+\frac{n}{\alpha}+c_{n} \ln n .
$$

COROLlary 4.3. For $p<1$ the iterates of $f$ are bounded by

$$
f^{n}(p)=\frac{\alpha p}{\alpha+p n+\alpha p c_{n} \ln n} .
$$

This proves the approximation part of the Theorem.

5. The case $d=\infty$. We include the limit case separately as it is of special importance and can be treated in a simpler way. Let

$$
G(y)=\frac{1}{f_{\infty}(1 / y)}=\frac{1}{1-\exp (-1 / y)}=\frac{\exp (1 / y)}{\exp (1 / y)-1} .
$$

Instead of analyzing $f_{\infty}(x)$ near 0 we can investigate $G(y)$ near $\infty$. Let

$$
H(y)=y(G(y)-y-1 / 2) \text {. }
$$

LEMMA 5.1. $\lim _{y \rightarrow 0^{+}} H(y)=0, \lim _{y \rightarrow \infty} H(y)=1 / 12$ and $H^{\prime}(y)>0$ for $0<y<\infty$. In other words, $H$ increases from 0 to $1 / 12$ as $y$ varies from 0 to $\infty$.

Proof. As $\lim _{y \rightarrow 0^{+}} \exp (-1 / y)=0, G(0)=1$ and $H(0)=0$. At $\infty$ first we calculate the asymptote $\lim _{y \rightarrow \infty} G(y) / y=1$ and $\lim _{y \rightarrow \infty} G(y)-y=1 / 2$. Next we return to the variable $x=1 / y$ and calculate the limit

$$
\lim _{x \rightarrow 0^{+}} \frac{1}{x}\left(\frac{1}{1-\exp (-x)}-\frac{1}{x}-\frac{1}{2}\right)=\lim _{x \rightarrow 0^{+}} \frac{2 x-(2+x)(1-\exp (-x))}{2 x^{2}(1-\exp (-x))}
$$

where it is easy to use the expansion (up to $x^{3}$ ) or de l'Hospital's rule. The monotonicity is as elementary but a bit more complicated because the tangency at infinity is of 6th order. For the record:

$$
H^{\prime}(y)=\frac{2 e^{1 / y}-y+e^{2 / y} y-4 y^{2}+8 e^{1 / y} y^{2}+4 e^{2 / y} y^{2}}{2\left(-1+e^{1 / y}\right)^{2} y},
$$


where the denominator is positive. We investigate the numerator after substituting $x=1 / y$ :

$$
N_{1}(x)=\frac{-4+8 e^{x}-4 e^{2 x}-x+e^{2 x} x+2 e^{x} x^{2}}{x^{2}},
$$

and again the denominator is positive and the value of the numerator at $x=0$ is 0 . It will be enough to prove that the numerator is an increasing function. After taking several derivatives and skipping obviously positive factors we obtain a sequence of functions which are 0 at $x=0$ :

$$
\begin{aligned}
& N_{2}(x)=N_{1}^{\prime}(x)=-1+e^{2 x}(-7+2 x)+2 e^{x}\left(4+2 x+x^{2}\right), \\
& N_{3}(x)=N_{2}^{\prime}(x) \cdot \exp (-x)=2\left(6+2 e^{x}(-3+x)+4 x+x^{2}\right), \\
& N_{4}(x)=N_{3}^{\prime}(x)=4\left(2+e^{x}(-2+x)+x\right), \\
& N_{5}(x)=N_{4}^{\prime}(x)=4+4 e^{x}(-1+x), \\
& N_{6}(x)=N_{5}^{\prime}(x)=4 e^{x} x .
\end{aligned}
$$

$N_{6}(x)>0$ hence for $x>0$ so is $N_{5}$, as $N_{5}(0)=0$, and going up we get $N_{1}(x)>0$, which proves that $H^{\prime}>0$ and so $H$ is increasing for $y>0$.

Corollary 5.2. For $1<y<\infty$,

$$
0.0819<a=\frac{3-e}{2(e-1)}=H(1)<H(y)<H(\infty)=\frac{1}{12}=b<0.0834,
$$

and therefore

$$
y+\frac{1}{2}+\frac{a}{y}<G(y)<y+\frac{1}{2}+\frac{b}{y} .
$$

One can also get a slightly worse estimate with $y=0.5$, namely $H(0.5)>$ 0.0782 .

Define

$$
S(y, n)=\sum_{k=0}^{n-1} \frac{2}{2 y+k} .
$$

Then $S(y, n)$ is of order $2 \ln \left(\frac{2 y+n}{2 y}\right)$. Let

$$
R(y, n)=\sum_{k=0}^{n-1} \frac{2}{2 y+k+2 b S(y, k)} .
$$

As $S(y, k) / k \rightarrow 0$ as $k \rightarrow \infty, R(y, n)$ is also of order $\ln \left(\frac{2 y+n}{2 y}\right)$ (up to a constant factor).

LEMmA 5.3. There are constants $0<A<B<\infty$ such that for all $y>1$ and all $n$,

$$
y+n / 2+A \ln n<G^{n}(y)<y+n / 2+B \ln n .
$$


Proof. First we find $G(y)>y+1 / 2$, hence $G^{n}(y)>y+n / 2$. We use this to estimate

$$
G^{k+1}(y)<G^{k}(y)+\frac{1}{2}+\frac{b}{G^{k}(y)}<G^{k}(y)+\frac{1}{2}+\frac{2 b}{2 y+k} .
$$

Hence by iterating we have $G^{n}(y)<y+n / 2+b S(y, n)$. Now we use this to obtain

$$
G^{k+1}(y)>G^{k}(y)+\frac{1}{2}+\frac{a}{G^{k}(y)}>G^{k}(y)+\frac{1}{2}+\frac{2 a}{2 y+k+2 b S(y, k)} .
$$

Summing up we get $G^{n}(y)>y+n / 2+2 a R(y, n)$. The logarithm estimate follows.

Corollary 5.4. There exist constants $0<A<B<\infty$ such that

$$
\frac{1}{n+1+B \ln n}<f_{\infty}^{n}(2)<\frac{1}{n+1+A \ln n}
$$

Proof. Set $x=2$, that is, $y=0.5$ and take the inverse of the estimate from the previous lemma.

6. Appendix: A motivational example. In this section we provide a heuristic reasoning, hence the formulations do not attempt to be rigorous.

Maximum weight problem. For $k$ sets $V_{1}, \ldots, V_{k}$ let $V$ be the set of all $k$ tuples $\left(v_{1}, \ldots, v_{k}\right)$ with $v_{i} \in V_{i}$ for $i=1, \ldots, k$. Given a feasibility condition on the subsets of $V$ and a positive weight function $w_{v}: V \rightarrow \mathbb{R}^{+}$choose a feasible subset of $k$-tuples with maximum sum of weights.

In the maximum $k$-dimensional axial assignment problem (also known as maximum $k$-dimensional matching problem) the set of $k$-tuples is feasible if no two $k$-tuples chosen have the same index in the same coordinate, i.e. if $v, v^{\prime}$ are in a feasible set then $v_{i} \neq v_{i}^{\prime}$ for $i=1, \ldots, k$. This problem has the following integer programming interpretation.

Let the cylinder $A(i, s)=\left\{\left(j_{1}, \ldots, j_{k}\right) \in V \mid j_{s}=i \in V_{s}\right\}$ be the set of all $k$-tuples with fixed $s$ th coordinate. Find

$$
\max \sum_{v \in V} w_{v} x_{v}
$$

under the conditions

$$
\begin{aligned}
& \sum_{v \in A(i, s)} x_{v}=1, \quad s=1, \ldots, k, i \in V_{s}, \\
& x_{v} \in\{0,1\}, \quad v \in V .
\end{aligned}
$$

The multi-dimensional assignment problems have various practical applications mainly in the fields of multitarget and multisensor tracking $[10,11]$. They are studied extensively in the operations research and theoretical computer science communities (see survey articles $[8,11]$ ). 
While the original problem (6.1)-(6.3) is computationally intractable (NP-hard) the linear relaxation of the problem obtained by relaxing the integrality constraint $x_{v} \in\{0,1\}$ to the constraint $0 \leq x_{v} \leq 1$ is just a linear programming problem and therefore can be solved efficiently.

Let $\left(x_{v}^{*}, v \in V\right)$ be the optimal solution of the linear programming relaxation for the problem (6.1)-(6.3). Obviously, $\sum_{v \in V} w_{v} x_{v}^{*}$ provides an upper bound for the optimal value of (6.1)-(6.3). The goal is to find an integer solution $\left(\bar{x}_{v}, v \in V\right)$ with $\sum_{v \in V} w_{v} \bar{x}_{v} \geq \rho * \sum_{v \in V} w_{v} x_{v}^{*}$. The number $\rho \in(0,1)$ is called the performance ratio. The field of design and analysis of approximation algorithms with bounded performance ratios is an active area of research [6].

Conjecture. There exists an algorithm which given a (so called) fractional solution $\left(x_{v}^{*}, v \in V\right)$ of the linear relaxation for the problem (6.1)-(6.3) finds an integer feasible solution $\left(\bar{x}_{v}, v \in V\right)$ such that

$$
\sum_{v \in V} w_{v} \bar{x}_{v} \geq p_{k-1} \cdot \sum_{v \in V} w_{v} x_{v}^{*} .
$$

Below we give a heuristic reasoning showing why this conjecture makes sense. We start by considering the optimal fractional solution $\left(x_{v}^{*}, v \in V\right)$. We generate an integral solution at random. At the first step we choose one $k$-tuple at random for each index $j \in V_{1}$ using the probability density function defined by $\left(x_{v}^{*}\right)$. The constraint (6.2) implies that $\sum_{v \in A(j, 1)} x_{v}=1$ for each $j \in V_{1}$. Therefore, at the end of the first step we choose a random set of $k$-tuples such that all constraints are satisfied for indices from $V_{1}$ and $\operatorname{Pr}\left(\bar{x}_{v}=1\right)=x_{v}^{*}=p_{0} x_{v}^{*}$.

At the second step, we consider the set of indices defined by $V_{2}$. For each $j \in V_{2}$ we might have a few $k$-tuples chosen at the previous step of the algorithm that have index $j$ as their second coordinate. We choose one of those $k$ tuples at random using the contention resolution process defined below.

Contention Resolution. There are $d$ players and one item. Each player $i$ requests the item independently at random with probability $p_{i}$. Let $A$ be a random set of players that request the item. Feige and Vondrak [3] suggested the scheme for allocating the item to one player only:

(1) If $A=\emptyset$ then do not allocate the item;

(2) If $|A|=1$ then allocate the item to player $k \in A$;

(3) If $|A|>1$ then allocate the item to player $k \in A$ with probability

$$
\left(\sum_{i \in\{A\} \backslash\{k\}} \frac{p_{i}}{|A|-1}+\sum_{i \notin\{A\}} \frac{p_{i}}{|A|}\right) / \sum_{i=1}^{d} p_{i} .
$$


Lemma 6.1 (Feige and Vondrak [3]). Conditioned on player $k$ requesting the item she obtains it with probability exactly

$$
\left.\rho=\frac{1-\prod_{i=1}^{d}\left(1-p_{i}\right)}{\sum_{i=1}^{d} p_{i}}{ }^{1}\right) .
$$

Assuming that each $k$-tuple having index $j$ as its second coordinate is chosen independently of any other such $k$-tuple (which is not generally true) we deduce therefore that the probability that a $k$-tuple $v$ with the second coordinate $j_{2}=j$ is still chosen after the second step is

$$
\begin{aligned}
\frac{1-\prod_{u \in V \mid j_{2}=j}\left(1-x_{u}^{*}\right)}{\sum_{u \in V \mid j_{2}=j} x_{u}^{*}} x_{v}^{*} & =\left(1-\prod_{u \in V \mid j_{2}=j}\left(1-x_{u}^{*}\right)\right) x_{v}^{*} \\
& \geq\left(1-\frac{1}{|A(j, 2)|}\right)^{|A(j, 2)|} x_{v}^{*} \\
& \geq\left(1-\frac{1}{|V|}\right)^{|V|} x_{v}^{*}=p_{1} x_{v}^{*} .
\end{aligned}
$$

Without loss of generality we assume that the probability of survival is exactly $p_{1} x_{v}^{*}$ since we can easily decrease it by killing a $k$-tuple $v$ with corresponding probability.

Assume that after $t$ steps we have an integer random solution $\bar{x}_{v}, v \in V$, such that all the constraints for the index sets $V_{1}, \ldots, V_{t}$ are satisfied and that $\operatorname{Pr}\left(\bar{x}_{v}=1\right)=p_{t-1} x_{v}^{*}$. We consider the index set $V_{t+1}$ and an index $j \in V_{t+1}$. Assuming that each $k$-tuple having index $j$ as its $(t+1)$ th coordinate is chosen independently of any other such $k$-tuple (again this is not generally true) and using the contention resolution algorithm defined above we infer that the probability that a $k$-tuple $v$ with the $(t+1)$ th coordinate $j_{t+1}=j$ is still chosen after the $(t+1)$ th step is

$$
\begin{aligned}
\left(\frac{1-\prod_{u \in V \mid j_{t+1}=j}\left(1-p_{t-1} x_{u}^{*}\right)}{\sum_{u \in V \mid j_{t+1}=j} p_{t-1} x_{u}^{*}}\right) p_{t-1} x_{v}^{*} & =\left(1-\prod_{u \in V \mid j_{t+1}=j}\left(1-p_{t-1} x_{u}^{*}\right)\right) x_{v}^{*} \\
& \geq\left(1-\frac{1}{|A(j, t+1)|}\right)^{|A(j, t+1)|} x_{v}^{*} \\
& \geq\left(1-\frac{1}{|V|}\right)^{|V|} x_{v}^{*}=p_{t} x_{v}^{*} .
\end{aligned}
$$

Again without loss of generality we assume that the probability of survival is exactly $p_{t} x_{v}^{*}$ since we can easily decrease it by killing a $k$-tuple $v$ with corresponding probability. After repeating the process for $k$ steps we obtain

$\left({ }^{1}\right)$ With $p_{i} \equiv p=1 / d$ we get our evolution $\rho=f_{d}(p)$. 
an integral feasible random solution $\left(\bar{x}_{v}, v \in V\right)$ such that

$$
E\left(\sum_{v \in V} w_{v} \bar{x}_{v}\right)=\sum_{v \in V} w_{v} \operatorname{Pr}\left(\bar{x}_{v}=1\right)=p_{k-1} \sum_{v \in V} w_{v} x_{v}^{*} .
$$

Although the analysis presented above does not work in the general case when the random events generated by the algorithm above might be dependent, we believe that there exists an appropriate modification of the algorithm that achieves the performance ratio of $p_{k-1}$.

Although the performance guarantee of $p_{k-1}$ of our method is a little worse than the performance guarantee of $2 /(k+1)$ of the best known approximation algorithm for the maximum $k$-dimensional axial assignment [2], our heuristic reasoning can be adapted to more general problems such as $d$-fold $k$-dimensional assignment problem [11]. Moreover, the known approximation algorithm is based on a local search framework that produces an algorithm with high running time, much slower than an algorithm based on linear programming.

\section{References}

[1] V. I. Arnold, Ordinary Differential Equations, MIT Press, 1978.

[2] P. Berman, A d/2 approximation for maximum weight independent set in $d$-claw free graphs, Nordic J. Comput. 7 (2000), 178-184.

[3] U. Feige and J. Vondrak, Approximation algorithms for allocation problems: Improving the factor of $1-1 / e$, in: FOCS 2006, 667-676.

[4] D. Gamarnik, T. Nowicki and G. Świrszcz, Invariant probability measures and dynamics of exponential linear type maps, Ergodic Theory Dynam. Systems 28 (2008), 1479-1495.

[5] - , - - , Maximum weight independent sets and matchings in sparse random graphs. Exact results using the local weak convergence method, Random Structures Algorithms 28 (2006), 76-106.

[6] T. F. Gonzalez (ed.), Handbook of Approximation Algorithms and Metaheuristics, Computer and Information Sci. Ser., Chapman \& Hall/CRC, Boca Raton, FL, 2007.

[7] L. Keen and R. Devaney (eds.), Complex Dynamics: twenty-five years after the appearance of the Mandelbrot set (Snowbird, UT, 2004), Contemp. Math. 396, Amer. Math. Soc., 2006.

[8] P. Pardalos and L. Pitsoulis, Quadratic and multidimensional assignment problems, in: Nonlinear Optimization and Related Topics (Erice, 1998), Appl. Optim. 36, Kluwer, Dordrecht, 2000, 235-256.

[9] H. Poincaré, Méthodes Nouvelles de la Mécanique Céleste, Vol. 2, Gauthier-Villars, Paris, 1893.

[10] A. Poore, Multidimensional assignment problems arising in multitarget and multisensor tracking, in: Nonlinear Assignment Problems, Combin. Optim. 7, Kluwer, Dordrecht, 2000, 13-38. 
[11] M. Queyranne and F. Spieksma, Multi-index transportation problems, in: The Encyclopedia of Optimization, C. Floudas and P. Pardalos (eds.), Kluwer, Dordrecht, 2001.

T. Nowicki, M. Sviridenko, G. Świrszcz, S. Winograd

IBM T. J. Watson Research Center

1101 Kitchawan Road

PO BOX 218

Yorktown Heights, NY 10598, U.S.A.

E-mail: tnowicki@us.ibm.com

sviri@us.ibm.com

swirszcz@us.ibm.com

swino@us.ibm.com

Received 16 January 2009;

in revised form 22 April 2009 